\title{
An Analysis on Correlation between the Curriculum and the Career of Students in Life Dance Department
}

\author{
Hyun Soon, Baek \\ Dance Department, Korea National Sport University \\ Yae Soon, Lee \\ Dance Department, Korea National Sport University
}

\begin{abstract}
I aim to discover that how greatly the curriculum of Life Dance department has had an effect on the career of students majoring in dance, with research objects being Life Dance department of two universities, located on the metropolitan area. The study method, called qualitative, includes some suggestions on the curriculum of the two and comparison, analysis of job-related materials; further, it involves discussion on the influence the curriculum has had on the career as dance trainers. The results from it are as follows; first, the curriculum of the department mostly consists of pure art such as Korean dance, modern dance, ballet, but has few creative life dance programs. Second, the employment into the art area has been more often than to the counterpart. Finally, it shows that a title of a department does not make a big difference and affect the employment rate. In conclusion, as the completion of the curriculum does not always guarantee a success, it is recommended that college students get some competent certificates related to dance.
\end{abstract}

Keywords: Life Dance Department, Curriculum, Career

\section{INTRODUCTION}

E. L. Tolbert, in Counseling for Career Development, has demonstrated that career has some meanings beyond the simple fact that a man becomes an employee, which illustrates that our life must be influenced by chosen jobs [1]. Jaechang Lee's study entitled "Application of career research programs" has showed that depending on the field we decide to work on, human beings are bound to be affected by many aspects of life, such as the opportunities, residence, the types of friendship, people's social or economic status, values, mental or physical health, and relationship among family members [2]. However, it is true that a lot of the students majoring in dance have difficulties in choosing a job; most of the curriculums in the universities are made up of Korean dance, Modern dance, and Ballet, aimed at the practical technique, which lowers the opportunities to become professional dancers or dance trainers. Fortunately, with the introduction of a pool system for dance instructors, there are said to be job openings for trainers in middle or high schools, but the number of people recruited is limited. Another challenge is that job applicants for the position should have a professional teaching certificate.

Considering the situation, the name of the department has been changed from Dance to Life Dance. The trial starts form the idea that it is possible to broaden access to jobs, by means

* Corresponding author. E-mail : back1006@hanmail.net Manuscript received Oct. 21, 2010 ; accepted Dec. 20, 2010 of the popularization of dance. Kyoungjoo Lee' research demonstrated that Life dance is the physical activity which people from all walks of life take part in, ranging from preschool children to the old; the dance performed for health improvement, beauty is meant by dance sport, jazz dance, aerobic dance and belly dance and also defined as informal, free, and active [3]. Therefore, as Heesun Lee suggests in her study on educational functions of dance [4], life dance can be called 'dance for all' or 'dance as a life-long activity'; the word, 'social dance' currently becomes synonymous with ' dance for all'. Hyeja Lim thus contends that Life dance is closely related to life-long education, learning society and can be defined as wholeness of dance, accompanying spontaneity of participants regardless of age and sex [5].

I am in need of research of the curriculum composition of Life Dance department, as well as the career of students in the dance section. The precedent studies, regarding the career, largely fall into three. For example, studies by Kunmi Lee, Younghee Kim and Myungjoo Kim [6]-[8] proved that one is that career guide affects choice of job, another is that the level of dance achievement does, and the other is that the earlier the students choose to advance into dance community, the higher the expectation of the success the greater part of the studies have been concentrated on one about thought, decision of career and dance itself." Unlike previous research, this paper explores the effects on the curriculum of Life Dance department has had on students' career, analysis of educational contents in the department, and the influence the contents have 
had on their career. Finally it finds out the problems and suggests the solutions to them.

\section{METHOD}

I focus on an analysis on correlation between the curriculum of Life Dance department and its career, with the subjects Life Dance department of two schools in the metropolitan area. The methodology, called qualitative, includes some suggestions on the curriculum of the two and comparison, analysis of jobrelated materials for the last three years; moreover, it covers discussion on an influence the curriculum has had on the career as dance trainers. $<$ Table $1>$ shows general characteristics of both universities; <Table 2> - <Table 9> illustrate each curriculum of both universities and finally <Table 10> indicates the career analysis on the graduates for the last three years.

Table 1. Characteristics of both universities

\begin{tabular}{|l|l|l|}
\hline University & $\begin{array}{l}\text { Total } \\
\text { (per grade) }\end{array}$ & $\begin{array}{l}\text { Major(The number of } \\
\text { students) }\end{array}$ \\
\hline A & 140 & $\begin{array}{l}\text { Korean Dance(18), } \\
\text { Modern Dance(12), } \\
\text { Ballet(10) }\end{array}$ \\
\hline B & 40 & $\begin{array}{l}\text { Korean Dance(14), } \\
\text { Modern Dance(13), } \\
\text { Ballet(13) }\end{array}$ \\
\hline
\end{tabular}

Table 2. Curriculum of university A(Freshman)

\begin{tabular}{|c|c|c|c|c|}
\hline \multirow{2}{*}{ Division } & \multirow{2}{*}{ Curriculum } & \multirow{2}{*}{ Hour } & \multicolumn{2}{|c|}{ Semester } \\
\hline & & & $1 \mathrm{st}$ & 2nd \\
\hline $\begin{array}{l}\text { (Major) } \\
\text { Theory } \\
\text { / elective }\end{array}$ & $\begin{array}{l}\text { Introduction } \\
\text { to Dance }\end{array}$ & 2 & 2 & \\
\hline $\begin{array}{l}\text { (Major ) } \\
\text { Theory } \\
\text { / elective }\end{array}$ & $\begin{array}{l}\text { Stage } \\
\text { Makeup }\end{array}$ & 2 & & 2 \\
\hline $\begin{array}{l}\text { (Major ) } \\
\text { Theory } \\
\text { / elective }\end{array}$ & $\begin{array}{l}\text { Modern } \\
\text { Dance I }\end{array}$ & 2 & 1 & \\
\hline $\begin{array}{l}\text { (Major ) } \\
\text { Theory } \\
\text { / elective }\end{array}$ & $\begin{array}{l}\text { Korean } \\
\text { Dance I }\end{array}$ & 2 & 1 & \\
\hline $\begin{array}{l}\text { (Major ) } \\
\text { Theory } \\
\text { / elective }\end{array}$ & Ballet I & 2 & 1 & \\
\hline $\begin{array}{l}\text { (Major ) } \\
\text { Theory } \\
\text { / elective }\end{array}$ & $\begin{array}{l}\text { Modern } \\
\text { Dance II }\end{array}$ & 2 & & 1 \\
\hline $\begin{array}{l}\text { (Major ) } \\
\text { Theory } \\
\text { / elective }\end{array}$ & $\begin{array}{l}\text { Korean } \\
\text { Dance II }\end{array}$ & 2 & & 1 \\
\hline $\begin{array}{l}\text { (Major ) } \\
\text { Theory } \\
\text { / elective }\end{array}$ & Ballet II & 2 & & 1 \\
\hline
\end{tabular}

\begin{tabular}{|c|c|c|c|}
\hline \multirow{3}{*}{$\begin{array}{l}\text { (Major ) } \\
\text { Theory } \\
\text { / elective }\end{array}$} & $\begin{array}{l}\text { Korean Dance } \\
\text { I,,II } \\
\text { (Basic) }\end{array}$ & \multirow[t]{3}{*}{4} & \multirow[t]{3}{*}{1} \\
\hline & $\begin{array}{l}\text { Modern Dance } \\
\text { I,II } \\
\text { (Basic) }\end{array}$ & & \\
\hline & $\begin{array}{l}\text { Choosing one } \\
\text { of Ballet } \\
\text { II(Basic) }\end{array}$ & & \\
\hline
\end{tabular}

Table 3. Curriculum of university A(Sophomore)

\begin{tabular}{|c|c|c|c|c|}
\hline \multirow{2}{*}{ Division } & \multirow{2}{*}{ Curriculum } & \multirow{2}{*}{ Hour } & \multicolumn{2}{|c|}{ Semester } \\
\hline & & & $1 \mathrm{st}$ & 2nd \\
\hline $\begin{array}{l}\text { (Major) } \\
\text { Theory } \\
\text { /elective }\end{array}$ & $\begin{array}{l}\text { Rhythm } \\
\text { Training }\end{array}$ & 2 & 2 & \\
\hline $\begin{array}{l}\text { (Major ) } \\
\text { theory } \\
\text { / elective }\end{array}$ & $\begin{array}{l}\text { Rhythm } \\
\text { Training } \\
\text { (in English) }\end{array}$ & 2 & 2 & \\
\hline $\begin{array}{l}\text { (Major ) } \\
\text { theory } \\
\text { / elective }\end{array}$ & $\begin{array}{l}\text { Folk } \\
\text { Dance }\end{array}$ & 2 & 2 & \\
\hline $\begin{array}{l}\text { (Major ) } \\
\text { theory } \\
\text { / elective }\end{array}$ & $\begin{array}{l}\text { Dance } \\
\text { Massage }\end{array}$ & 2 & 2 & \\
\hline $\begin{array}{l}\text { (Major ) } \\
\text { Theory } \\
\text { / elective }\end{array}$ & $\begin{array}{l}\text { Oriental Dance } \\
\text { History }\end{array}$ & 2 & & 2 \\
\hline $\begin{array}{l}\text { (Major ) } \\
\text { theory } \\
\text { / elective }\end{array}$ & $\begin{array}{l}\text { Sport } \\
\text { History }\end{array}$ & 3 & & 3 \\
\hline $\begin{array}{l}\text { (Major ) } \\
\text { Theory } \\
\text { / elective }\end{array}$ & $\begin{array}{ll}\text { Principles } & \\
\text { of } & \text { Physical } \\
\text { Education } & \end{array}$ & 3 & & 3 \\
\hline $\begin{array}{l}\text { (Major ) } \\
\text { Theory } \\
\text { / elective }\end{array}$ & $\begin{array}{l}\text { Western Dance } \\
\text { History }\end{array}$ & 2 & 2 & \\
\hline $\begin{array}{l}\text { (Major ) } \\
\text { Theory } \\
\text { / elective }\end{array}$ & $\begin{array}{l}\text { Human } \\
\text { Anatomy }\end{array}$ & 2 & 2 & \\
\hline $\begin{array}{l}\text { (Major ) } \\
\text { Practice } \\
\text { / elective }\end{array}$ & $\begin{array}{l}\text { Korean } \\
\text { Dance } \\
\text { III,IV }\end{array}$ & 4 & 1 & \\
\hline $\begin{array}{l}\text { (Major ) } \\
\text { practice } \\
\text { / elective }\end{array}$ & $\begin{array}{l}\text { Modern } \\
\text { Dance } \\
\text { III,IV } \\
\end{array}$ & 4 & 1 & 1 \\
\hline $\begin{array}{l}\text { (Major ) } \\
\text { Practice } \\
\text { / elective } \\
\end{array}$ & $\begin{array}{l}\text { Ballet } \\
\text { III, IV }\end{array}$ & 4 & 1 & 1 \\
\hline $\begin{array}{l}\text { (Major) } \\
\text { Practice }\end{array}$ & $\begin{array}{l}\text { Korean } \\
\text { Dance } \\
\text { (Basic) }\end{array}$ & 4 & 1 & 1 \\
\hline
\end{tabular}




\begin{tabular}{|l|l|l|l|l|}
\hline / elective & $\begin{array}{l}\text { Modern } \\
\text { Dance } \\
\text { (Basic) }\end{array}$ & \multirow{2}{*}{} & & \\
\cline { 2 - 4 } & $\begin{array}{l}\text { Choosing } \\
\text { one of Ballet I,II } \\
\text { (Basic) }\end{array}$ & & \\
\hline $\begin{array}{l}\text { (Major ) } \\
\text { Practice } \\
\text { / elective }\end{array}$ & Make-up & 2 & & 1 \\
\hline
\end{tabular}

\begin{tabular}{|l|l|l|l|l|}
\hline & $\begin{array}{l}\text { Choosing } \\
\text { one of } \\
\text { Ballet Workshop } \\
\text { I,II }\end{array}$ & & & \\
\hline $\begin{array}{l}\text { (Major ) } \\
\text { Practice } \\
\text { / elective }\end{array}$ & $\begin{array}{l}\text { Jazz I, } \\
\text { Dance Sport I }\end{array}$ & 2 & 1 \\
\hline $\begin{array}{l}\text { (Major ) } \\
\text { Practice } \\
\text { /elective }\end{array}$ & $\begin{array}{l}\text { Yoga } \\
\text { I, II }\end{array}$ & 4 & 1 & 1 \\
\hline
\end{tabular}

Table 4. Curriculum of university A(Junior)

\begin{tabular}{|c|c|c|c|c|}
\hline \multirow{2}{*}{ Division } & \multirow{2}{*}{ Curriculum } & \multirow{2}{*}{ Hour } & \multicolumn{2}{|c|}{ Semester } \\
\hline & & & $1 \mathrm{st}$ & 2nd \\
\hline $\begin{array}{l}\text { (Major) } \\
\text { theory } \\
\text { / elective }\end{array}$ & $\begin{array}{l}\text { Sports } \\
\text { Psychology }\end{array}$ & 3 & 3 & \\
\hline $\begin{array}{l}\text { (Major ) } \\
\text { theory } \\
\text { / elective }\end{array}$ & $\begin{array}{l}\text { Exercise } \\
\text { Physiology }\end{array}$ & 3 & & 3 \\
\hline $\begin{array}{l}\text { (Major ) } \\
\text { theory } \\
\text { / elective }\end{array}$ & $\begin{array}{l}\text { Dance } \\
\text { Sociology }\end{array}$ & 3 & 3 & \\
\hline $\begin{array}{l}\text { (Major ) } \\
\text { theory } \\
\text { / elective }\end{array}$ & $\begin{array}{l}\text { Analysis and } \\
\text { Notation of Dance } \\
\text { Movement }\end{array}$ & 2 & 2 & \\
\hline $\begin{array}{l}\text { (Major ) } \\
\text { theory } \\
\text { / elective }\end{array}$ & $\begin{array}{l}\text { Dance } \\
\text { Nutrition }\end{array}$ & 2 & 2 & \\
\hline $\begin{array}{l}\text { (Major) } \\
\text { theory } \\
\text { / elective }\end{array}$ & $\begin{array}{l}\text { Dance } \\
\text { Therapy }\end{array}$ & 2 & 2 & \\
\hline $\begin{array}{l}\text { (Major ) } \\
\text { theory } \\
\text { / elective }\end{array}$ & $\begin{array}{l}\text { Statistics and } \\
\text { Research } \\
\text { Method in Sport }\end{array}$ & 3 & & 3 \\
\hline $\begin{array}{l}\text { (Major ) } \\
\text { theory } \\
\text { / elective }\end{array}$ & $\begin{array}{l}\text { Stage } \\
\text { Theory }\end{array}$ & 2 & & 2 \\
\hline $\begin{array}{l}\text { (Major ) } \\
\text { Theory } \\
\text { / elective }\end{array}$ & $\begin{array}{l}\text { Dance } \\
\text { Composition }\end{array}$ & 2 & & 2 \\
\hline $\begin{array}{l}\text { (Major ) } \\
\text { Theory } \\
\text { / elective }\end{array}$ & $\begin{array}{l}\text { Dance } \\
\text { Music }\end{array}$ & 2 & & 2 \\
\hline $\begin{array}{l}\text { (Major ) } \\
\text { theory } \\
\text { / elective }\end{array}$ & $\begin{array}{l}\text { Introduction } \\
\text { to Costumes }\end{array}$ & 2 & & 2 \\
\hline $\begin{array}{l}\text { (Major ) } \\
\text { Practice } \\
\text { / elective }\end{array}$ & $\begin{array}{l}\text { Traditional } \\
\text { Dance I }\end{array}$ & 2 & & 1 \\
\hline \multirow{2}{*}{$\begin{array}{l}\text { (Major ) } \\
\text { Practice } \\
\text { / elective }\end{array}$} & $\begin{array}{l}\text { Korean } \\
\text { Dance } \\
\text { Workshop } \\
\text { I, II }\end{array}$ & \multirow{2}{*}{8} & \multirow{2}{*}{2} & \multirow{2}{*}{2} \\
\hline & $\begin{array}{l}\text { Modern } \\
\text { Dance } \\
\text { Workshop } \\
\text { I, II }\end{array}$ & & & \\
\hline
\end{tabular}

Table 5. Curriculum of university A(Senior)

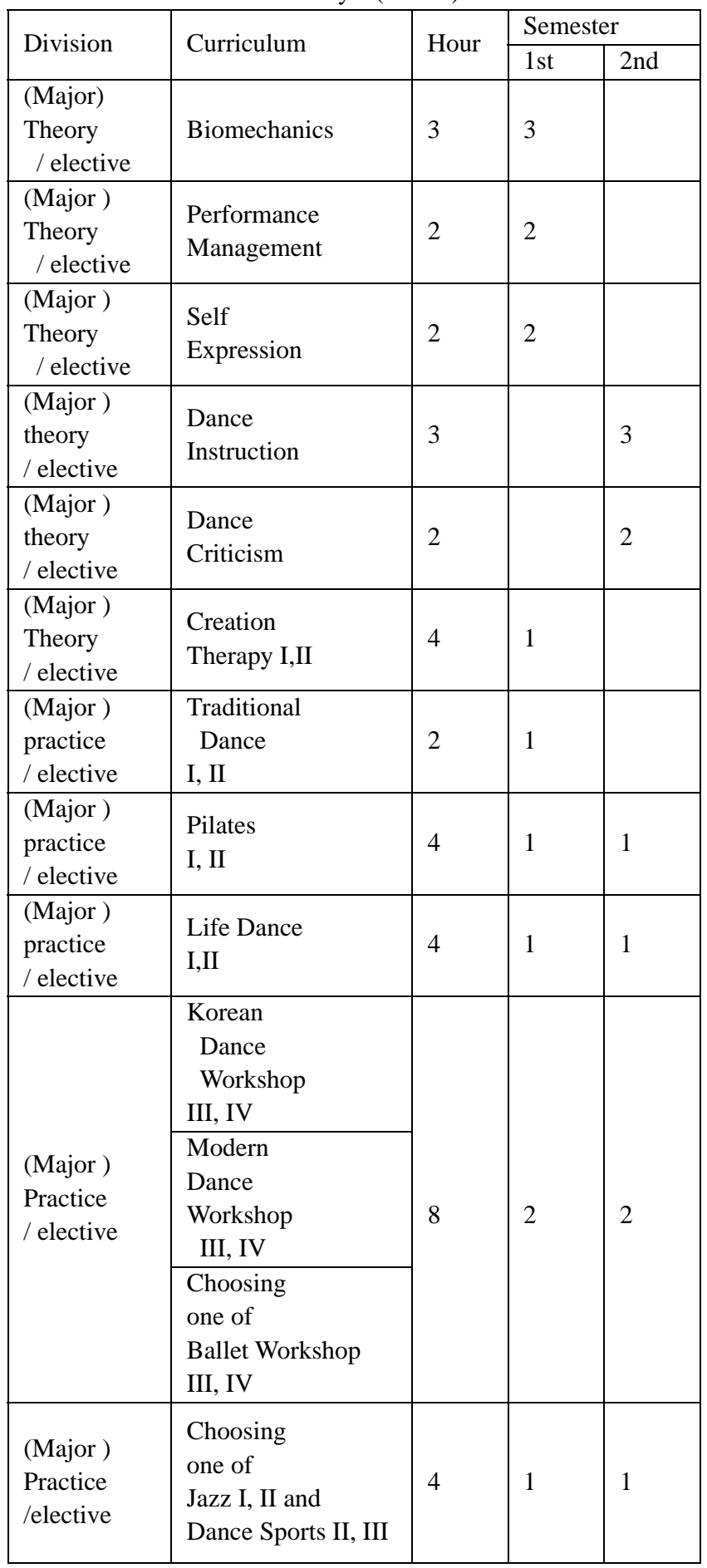


Table 6. Curriculum of university B(Freshman)

\begin{tabular}{|c|c|c|c|c|}
\hline \multirow{2}{*}{ Division } & \multirow{2}{*}{ Curriculum } & \multirow{2}{*}{ Hour } & \multicolumn{2}{|c|}{ Semester } \\
\hline & & & $1 \mathrm{st}$ & 2nd \\
\hline $\begin{array}{l}\text { Required } \\
\text { (Basic) }\end{array}$ & $\begin{array}{l}\text { Human } \\
\text { Anatomy }\end{array}$ & 3 & 0 & \\
\hline \multirow{3}{*}{$\begin{array}{l}\text { Required } \\
\text { (Basic) }\end{array}$} & $\begin{array}{l}\text { Korean } \\
\text { Dance I }\end{array}$ & \multirow{3}{*}{1} & \multirow{3}{*}{0} & \\
\hline & $\begin{array}{l}\text { Modern } \\
\text { Dance I }\end{array}$ & & & \\
\hline & Ballet I & & & \\
\hline $\begin{array}{l}\text { Required } \\
\text { (Basic) }\end{array}$ & $\begin{array}{l}\text { Major Practice } \\
\text { of Dance I }\end{array}$ & 2 & 0 & \\
\hline $\begin{array}{l}\text { Required } \\
\text { (Basic) }\end{array}$ & $\begin{array}{l}\text { English } \\
\text { Communication }\end{array}$ & 2 & 0 & \\
\hline $\begin{array}{l}\text { Required } \\
\text { (Basic) }\end{array}$ & $\begin{array}{l}\text { Practical } \\
\text { English } \\
\text { Conversation I }\end{array}$ & 3 & 0 & \\
\hline $\begin{array}{l}\text { Required } \\
\text { (Basic) }\end{array}$ & $\begin{array}{l}\text { Seminar } \\
\text { for Freshmen }\end{array}$ & 1 & 0 & \\
\hline \multirow{3}{*}{$\begin{array}{l}\text { Required } \\
\text { (Basic) }\end{array}$} & $\begin{array}{l}\text { Korean } \\
\text { Dance II }\end{array}$ & \multirow{3}{*}{1} & & \multirow{3}{*}{0} \\
\hline & $\begin{array}{l}\text { Modern } \\
\text { Dance II }\end{array}$ & & & \\
\hline & Ballet II & & & \\
\hline $\begin{array}{c}\text { Required } \\
\text { (Basic) }\end{array}$ & $\begin{array}{l}\text { Korean } \\
\text { Speaking } \\
\text { and Writing } \\
\end{array}$ & 3 & & 0 \\
\hline $\begin{array}{l}\text { Required } \\
\text { (Basic) }\end{array}$ & $\begin{array}{l}\text { Art } \\
\text { Theory }\end{array}$ & 3 & & 0 \\
\hline $\begin{array}{l}\text { Required } \\
\text { (Basic) }\end{array}$ & $\begin{array}{l}\text { Creative } \\
\text { English } \\
\text { Reading }\end{array}$ & 2 & & 0 \\
\hline $\begin{array}{l}\text { Required } \\
\text { (Basic) }\end{array}$ & $\begin{array}{l}\text { Major } \\
\text { Practice } \\
\text { of Dance II }\end{array}$ & 2 & & 0 \\
\hline $\begin{array}{l}\text { Required } \\
\text { (Basic) }\end{array}$ & $\begin{array}{l}\text { Philosophical } \\
\text { Understanding } \\
\text { of Science and } \\
\text { Technology }\end{array}$ & 2 & & 0 \\
\hline
\end{tabular}

Table 7. Curriculum of university B(Sophomore)

\begin{tabular}{|l|l|l|l|l|}
\hline \multirow{2}{*}{ Division } & Curriculum & Hour & \multicolumn{2}{|l|}{ Semester } \\
\cline { 4 - 5 } & & 1 st & 2nd \\
\hline $\begin{array}{l}\text { Intensive } \\
\text { (Major) }\end{array}$ & $\begin{array}{l}\text { Analysis } \\
\text { on Computer } \\
\text { Movement }\end{array}$ & 3 & 0 & \\
\hline $\begin{array}{l}\text { Intensive } \\
\text { (Major) }\end{array}$ & $\begin{array}{l}\text { Dance } \\
\text { Production }\end{array}$ & 3 & 0 & \\
\hline $\begin{array}{l}\text { Core ( } \\
\text { Major) }\end{array}$ & $\begin{array}{l}\text { Life } \\
\text { Dance II }\end{array}$ & 1 & 0 & \\
\hline $\begin{array}{l}\text { Core } \\
\text { (Major) }\end{array}$ & $\begin{array}{l}\text { Workshop } \\
\text { for Dance } \\
\text { Practice III }\end{array}$ & 1 & 0 & \\
\hline $\begin{array}{l}\text { Core } \\
\text { (Major) }\end{array}$ & Choreography III & 2 & 0 & \\
\hline
\end{tabular}

\begin{tabular}{|c|c|c|c|c|}
\hline $\begin{array}{l}\text { Core } \\
\text { (Major) }\end{array}$ & $\begin{array}{l}\text { Educational } \\
\text { Theory } \\
\text { of Dance }\end{array}$ & 3 & 0 & \\
\hline $\begin{array}{l}\text { Core } \\
\text { (Major) }\end{array}$ & $\begin{array}{l}\text { Major Practice } \\
\text { of Dance V }\end{array}$ & 2 & 0 & \\
\hline $\begin{array}{l}\text { Core } \\
\text { (Major) }\end{array}$ & $\begin{array}{l}\text { Aesthetics } \\
\text { of Dance }\end{array}$ & 3 & 0 & \\
\hline $\begin{array}{l}\text { Intensive } \\
\text { (Major) }\end{array}$ & Pilates & 3 & & 0 \\
\hline $\begin{array}{l}\text { Intensive } \\
\text { (Major) }\end{array}$ & Gyrotonic & 3 & & 0 \\
\hline $\begin{array}{l}\text { Intensive } \\
\text { (Major) }\end{array}$ & $\begin{array}{l}\text { Computer } \\
\text { Dance } \\
\text { Application }\end{array}$ & 1 & & 0 \\
\hline $\begin{array}{l}\text { Intensive } \\
\text { (Major) }\end{array}$ & $\begin{array}{l}\text { Teaching } \\
\text { method } \\
\text { of Dance }\end{array}$ & 3 & & 0 \\
\hline $\begin{array}{l}\text { Core } \\
\text { (Major) }\end{array}$ & $\begin{array}{l}\text { Workshop } \\
\text { for Dance } \\
\text { Practice IV }\end{array}$ & 1 & & 0 \\
\hline $\begin{array}{l}\text { Core } \\
\text { (Major) }\end{array}$ & $\begin{array}{l}\text { Life } \\
\text { Dance III }\end{array}$ & 1 & & 0 \\
\hline $\begin{array}{l}\text { Core } \\
\text { (Major) }\end{array}$ & Choreography IV & 2 & & 0 \\
\hline $\begin{array}{l}\text { Core } \\
\text { (Major) }\end{array}$ & $\begin{array}{l}\text { Major Practice of } \\
\text { Dance VI }\end{array}$ & 2 & & 0 \\
\hline $\begin{array}{l}\text { Core } \\
\text { (Major) }\end{array}$ & Dance Creation & 3 & & 0 \\
\hline
\end{tabular}

Table 8. Curriculum of university B(Junior)

\begin{tabular}{|c|c|c|c|c|}
\hline \multirow{2}{*}{ Division } & \multirow{2}{*}{ Curriculum } & \multirow{2}{*}{ Hour } & \multicolumn{2}{|c|}{ Semester } \\
\hline & & & $1 \mathrm{st}$ & 2nd \\
\hline $\begin{array}{r}\text { Intensive } \\
\text { (Major) }\end{array}$ & $\begin{array}{l}\text { Dance } \\
\text { Sports I }\end{array}$ & 1 & 0 & \\
\hline $\begin{array}{l}\text { Intensive } \\
\text { (Major) }\end{array}$ & Jazz I & 1 & 0 & \\
\hline $\begin{array}{l}\text { Intensive } \\
\text { (Major) }\end{array}$ & $\begin{array}{l}\text { Percussion } \\
\text { Instrument } \\
\text { Practice I }\end{array}$ & 1 & 0 & \\
\hline $\begin{array}{l}\text { Intensive } \\
\text { (Major) }\end{array}$ & $\begin{array}{l}\text { Dance } \\
\text { Composition }\end{array}$ & 3 & 0 & \\
\hline $\begin{array}{l}\text { Core } \\
\text { (Major) }\end{array}$ & $\begin{array}{l}\text { Workshop } \\
\text { for } \\
\text { Dance } \\
\text { Practice }\end{array}$ & 1 & 0 & \\
\hline $\begin{array}{l}\text { Core } \\
\text { (Major) }\end{array}$ & Choreography I & 2 & 0 & \\
\hline $\begin{array}{l}\text { Core } \\
\text { (Major) }\end{array}$ & $\begin{array}{l}\text { History of } \\
\text { Western } \\
\text { Dance }\end{array}$ & 3 & 0 & \\
\hline $\begin{array}{l}\text { Core } \\
\text { (Major) }\end{array}$ & $\begin{array}{l}\text { History of } \\
\text { Korean } \\
\text { Dance }\end{array}$ & 3 & 0 & \\
\hline $\begin{array}{l}\text { Core } \\
\text { (Major) }\end{array}$ & $\begin{array}{l}\text { Function } \\
\text { of Dance }\end{array}$ & 3 & 0 & \\
\hline
\end{tabular}




\begin{tabular}{|c|c|c|c|c|}
\hline $\begin{array}{l}\text { Core } \\
\text { (Major) }\end{array}$ & $\begin{array}{l}\text { Major } \\
\text { Practice } \\
\text { of Dance III }\end{array}$ & 2 & 0 & \\
\hline $\begin{array}{l}\text { Intensive } \\
\text { (Major) }\end{array}$ & $\begin{array}{l}\text { Dance } \\
\text { Sports II }\end{array}$ & 1 & & 0 \\
\hline $\begin{array}{l}\text { Intensive } \\
\text { (Major) }\end{array}$ & Jazz II & 1 & & 0 \\
\hline $\begin{array}{r}\text { Intensive } \\
\text { (Major) }\end{array}$ & $\begin{array}{l}\text { Design } \\
\text { of Digital } \\
\text { Dance } \\
\text { Image }\end{array}$ & 3 & & 0 \\
\hline $\begin{array}{l}\text { Intensive } \\
\text { (Major) }\end{array}$ & $\begin{array}{l}\text { Percussion } \\
\text { Instrument } \\
\text { Practice II }\end{array}$ & 1 & & 0 \\
\hline $\begin{array}{l}\text { Intensive } \\
\text { (Major) }\end{array}$ & $\begin{array}{l}\text { Dance } \\
\text { Repertory }\end{array}$ & 3 & & 0 \\
\hline $\begin{array}{l}\text { Core } \\
\text { (Major) }\end{array}$ & $\begin{array}{l}\text { Workshop } \\
\text { for Dance } \\
\text { Practice }\end{array}$ & 1 & & 0 \\
\hline $\begin{array}{l}\text { Core } \\
\text { (Major) }\end{array}$ & $\begin{array}{l}\text { Life } \\
\text { Dance I }\end{array}$ & 1 & & 0 \\
\hline $\begin{array}{l}\text { Core } \\
\text { (Major) }\end{array}$ & Choreography II & 2 & & 0 \\
\hline $\begin{array}{l}\text { Core } \\
\text { (Major) }\end{array}$ & $\begin{array}{l}\text { Dance } \\
\text { Psychology }\end{array}$ & 3 & & 0 \\
\hline $\begin{array}{l}\text { Core } \\
\text { (Major) }\end{array}$ & $\begin{array}{l}\text { Major } \\
\text { Practice } \\
\text { of Dance IV }\end{array}$ & 2 & & 0 \\
\hline
\end{tabular}

\begin{tabular}{|l|l|l|l|l|}
\hline $\begin{array}{l}\text { Core } \\
\text { (Major) }\end{array}$ & Choreography VI & 2 & 0 \\
\hline $\begin{array}{l}\text { Core } \\
\text { (Major) }\end{array}$ & $\begin{array}{l}\text { Major } \\
\text { practice } \\
\text { of Dance VIII }\end{array}$ & 2 & 0 \\
\hline
\end{tabular}

Table 10. Career analysis (from 2008 to 2010)

\begin{tabular}{|c|c|c|}
\hline & University A & University B \\
\hline 2008 & $\begin{array}{l}\text { Graduates total: } 25 \\
\text { - full time job (4) } \\
\text { - temporary job (14) } \\
\text { - graduate school (7) } \\
\text {-Relation to major } \\
\text { (21) } \\
\text {-No relation to major } \\
\text { (4) } \\
\text {-Employment to life } \\
\text { dance field (2) } \\
\text {-Employment to } \\
\text { academies (12) }\end{array}$ & $\begin{array}{l}\text { Graduates total: } 39 \\
\text { - full time job (6) } \\
\text { - temporary job (28) } \\
\text { - graduate school (5) } \\
\text {-Relation to major } \\
\text { (34) } \\
\text {-No relation to major } \\
\text { (5) } \\
\text {-Employment to life } \\
\text { dance field (8) } \\
\text {-Employment to } \\
\text { academies (12) }\end{array}$ \\
\hline 2009 & $\begin{array}{l}\text { Graduates total: } 29 \\
\text { - full time job (7) } \\
\text { - temporary job (14) } \\
\text { - graduate school (8) } \\
\text {-Relation to major } \\
(26) \\
\text {-No relation to major } \\
\text { (3) } \\
\text {-Employment to life } \\
\text { dance field (9) } \\
\text {-Employment to } \\
\text { academies (9) }\end{array}$ & $\begin{array}{l}\text { Graduates total: } 28 \\
\text { - full time job (2) } \\
\text { - temporary job (16) } \\
\text { - graduate school (10) } \\
\text { Relation to major } \\
\text { (23) } \\
\text { No relation to major } \\
\text { (5) } \\
\text { Employment to life } \\
\text { dance field (3) } \\
\text { Employment } \\
\text { academies (3) }\end{array}$ \\
\hline 2010 & $\begin{array}{l}\text { Graduates total: } 24 \\
\text { - full time job (6) } \\
\text { - temporary job (14) } \\
\text { - graduate school (4) } \\
\text {-Relation to major } \\
\text { (19) } \\
\text {-No relation to major } \\
\text { (5) } \\
\text {-Employment to life }\end{array}$ & $\begin{array}{l}\text { Graduates total: } 20 \\
\text { - full time job (7) } \\
\text { - temporary job (8) } \\
\text { - graduate school (5) } \\
\text {-Relation to major } \\
\text { (15) } \\
\text {-No relation to major } \\
\text { (5 } \\
\text {-Employment to life }\end{array}$ \\
\hline
\end{tabular}

Table 9. Curriculum of university B(Senior)

\begin{tabular}{|c|c|c|c|c|}
\hline \multirow{2}{*}{ Division } & \multirow{2}{*}{ Curriculum } & \multirow{2}{*}{ Hour } & \multicolumn{2}{|c|}{ semester } \\
\hline & & & 1st & 2nd \\
\hline $\begin{array}{l}\text { Intensive } \\
\text { (Major) }\end{array}$ & $\begin{array}{l}\text { Computer } \\
\text { Dance } \\
\text { Application }\end{array}$ & 1 & 0 & \\
\hline $\begin{array}{l}\text { Core } \\
\text { (Major) }\end{array}$ & $\begin{array}{l}\text { Workshop } \\
\text { for } \\
\text { Dance } \\
\text { Practice V }\end{array}$ & 1 & 0 & \\
\hline $\begin{array}{l}\text { Core } \\
\text { (Major) }\end{array}$ & $\begin{array}{l}\text { Life } \\
\text { Dance } \\
\text { IV }\end{array}$ & 1 & 0 & \\
\hline $\begin{array}{l}\text { Core } \\
\text { (Major) }\end{array}$ & $\begin{array}{l}\text { Choreography } \\
\text { V }\end{array}$ & 2 & 0 & \\
\hline $\begin{array}{l}\text { Core } \\
\text { (Major) }\end{array}$ & $\begin{array}{l}\text { Dance } \\
\text { Sociology }\end{array}$ & 3 & 0 & \\
\hline $\begin{array}{l}\text { Core } \\
\text { (Major) }\end{array}$ & $\begin{array}{l}\text { Dance } \\
\text { Criticism }\end{array}$ & 3 & 0 & \\
\hline $\begin{array}{l}\text { Core } \\
\text { (Major) }\end{array}$ & $\begin{array}{l}\text { Major } \\
\text { practice } \\
\text { of Dance } \\
\text { VII }\end{array}$ & 2 & 0 & \\
\hline $\begin{array}{l}\text { Core } \\
\text { (Major) }\end{array}$ & $\begin{array}{l}\text { Life } \\
\text { Dance V }\end{array}$ & 1 & & 0 \\
\hline $\begin{array}{l}\text { Core } \\
\text { (Major) }\end{array}$ & $\begin{array}{l}\text { Workshop } \\
\text { for Dance } \\
\text { Practice VI }\end{array}$ & 1 & & 0 \\
\hline
\end{tabular}




\begin{tabular}{|l|l|l|}
\hline & $\begin{array}{l}\text { dance field (3) } \\
\text {-Employment } \\
\text { academies (11) }\end{array} \quad$ to & $\begin{array}{l}\text { dance field (0) } \\
\text {-Employment } \\
\text { academies (1) }\end{array} \quad$ to \\
\hline
\end{tabular}

\section{RESULT}

\subsection{An Analysis on Correlation between the Curriculum and Life Dance}

According to each grade, the curriculum of Life Dance department can be divided into four: first, pure art such as Korean dance, modern dance and ballet. Second, teaching parts, like dance theory, life dance, rhythm or percussion music, choreography or creation, planning and management. Third, the field of dance staff which covers make-up, costume, fine art, and image. Last, sport area. Most of the time is being spent in learning the domain of pure art, such as Korean dance, Modern dance and Ballet, in both universities. When it comes to school hours, university A has 18 and university B has 18 for 4 years. In case of $\mathrm{A}$, the classes are largely deployed in junior, senior grade, but B's hours are evenly done every grade, except freshmen.

\subsection{An Analysis on Correlation between the Curriculum and the Career}

As < Table 10> suggests above, the number of employees in dance institutes, involved in pure art is much more than that of life dance concerned; university A has 32 people who advanced into dance academies to teach Korean dance, ballet and 14 people who became life dance trainers to coach future dancers in Yoga, Pilates. On the contrary, university B has 16 employees in the area of pure art and 11 in the other part. As shown in $<$ Table $10>$, more than $90 \%$ of the employed made a decision to have major-related job; in case of A, 73 of 78 students (93.6\%) for the last three succeeded in being in a major-relevant position, including graduation school and in case of B, 82 of 87 students (83\%) did so. Unfortunately, $<$ Table 10> illustrates that most of the employment belong to not a full-time job, but a part-time, which makes job applicants consider it difficult to have a stable job.

\section{3 An Analysis on Correlation between the Department and the Career}

To give a department a name is of great importance; changing Dance to Life Dance department should encourage the college students to choose the career to the newly-named dance field. However, only because the number of life dance curriculums is a little more than that of the old one, it does not make a sense to name the new one Life Dance department. Both institutions has more curricula of pure art, like Korean dance, Modern Dance, and Ballet than those of life dance; the number of the employed concerned with the former is much more than it of the latter. University A has a lot of sport-related subjects, such as Sport History, Human Anatomy, Sport psychology, and Exercise Physiology, but university B has many dance programs combined to computer, like Korean Speaking and Writing,
Creative English Reading, Philosophical Understanding of Science and Technology, Analysis on Computer Movement, Computer Dance Application and Design of Digital Dance image. For college students who might think it difficult to have a grasp of the classes, all courses but ones in first grade include the subject of Choreography, which makes it possible for them to compose the dance, apply it to the computer, image and further to communicate with each other. Nevertheless, this fact does not have a positive effect on their employment.

\section{CONCLUSION}

What we are learning in the universities is said to be very critical, as it greatly influences our career. It has been believed that a greater part of college students begins to research their career, when they are senior. There are some factors affecting career search, decision; for example, relevant studies demonstrated that there are identity of career, personality, psychological independence from parents, family's social support and efficacy of career decision [10]-[13]. According to Jinsook Seo, the career research covers searching for self, finding out information of the world, and acquiring the basic knowledge for employment.

Learnt professional knowledge and acquired practical skill in the universities can be regarded as basic ability that job seekers must have to be employed. As mentioned above, what we are learning in the universities matters in choosing the career. The conclusions drawn from learning 4-year content of Life Dance department are as follows; first, the curriculum of the department generally consists of Korean dance, Modern dance, Ballet and has few creative life dance programs. Second, the employment to pure art has been more often than to the counterpart, which indicates that a specific curriculum seems to be irrelevant to employment. That is, it is required to check out whether Choreography classes involve the contents related to the development of life dance or leads to the employment of the domain of the computer dance application. Finally, it shows that a name of a department does not make a big difference and affect the employment rate very much.

\section{REFERENCE}

[1] Tolbert. E. L, Counseling for Career Development (2nd ed), Houghton Miffin, Boston .1980.

[2] Jaechang Lee, "Application of career research programs", Research of college life, no. 10, Institute of College Life in Hanyang University, 1992, pp19-36.

[3] Kyoungjoo, Lee, A social, psychological analysis on the participation in life dance, doctoral dissertation, Konkuk University, 2010.

[4] Heesun, Lee, "A study on educational functions of dance", Journal of Korean Dance Education, no. 5, 1995, pp65-73.

[5] Hyeja, Lim, Life dance programs' influence on life satisfaction and psychological change, doctoral dissertation, Kyungpook University, 1996. 
[6] Kunmi Lee, Correlation among the efficacy of career choice, research, and the types of career choice depending on career guidance on college students majoring dance, doctoral dissertation, Hanyang University, 2008.

[7] Younghee Kim, The adaptation to studies, attitude of career choice affected by the achievement of students majoring dance, doctoral dissertation, Dankook University, 2007.

[8] Myungjoo Kim, “A study on dance majoring college students' career awareness and choice”, Journal of Korean dance institute, no. 58, Korean Dance Institute, 2009, pp19-39.

[9] Blustein. D. L, "The role of career exploration in the career decision making of college students”, Journal of College Student Development, no. 30, pp111-117, 1989.

[10] Aeri Cho, Correlation among the standard of college students' career choice, research, and 5 personality factors, master's thesis, Seoul University, 1999.

[11] Lee. H. Y and Hughey. K. F, "The relationship of Psychological separation and parental attachment to the career maturity of college freshmen from intact families”, Journal of Career Development, vol. 27, no.4, 2001, pp279-293.

[12] Hyunjoo Lee(2001), “A study on cause -effect relationship between college students' social, psychological characteristic and career research" , Korean Psychology Research , no.15, pp257-280, 2001

[13] Betz. N. E and Hackett. G, “Application of self-efficacy theory to understanding career choice behavior”, Journal of Social and Clinical Psychology, no. 4, 1986, pp279289.

[14] Dukyeon Lee, Relationship among some variables affecting career research - an analysis on covariate structure depending on the standard of career choice-, doctoral thesis, Chunnam University, 2004, P16.

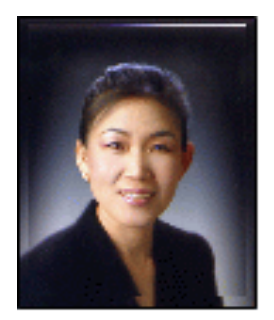

\section{Hyun Soon Baek}

She received the B.S. in dance from Catholic University of Daegu, Korea, in 1981, the M.S. in dance education from Ewha Womans University, Korea, in 1984, and a Ph. D in dance from Kyonggi University, Korea, in 1999. Since then, she has been with Life Dance Department, Korea National Sport University. Her main research interests include dance philosophy and dance history.

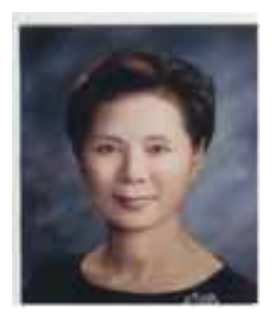

\section{Yae Soon, Lee}

She received the B.S. in the dance from Ewha Womans University, Korea, in 1968, the M.S. in modern dance from Dong-A University, Korea, in 1976 and Ph. D (honorary) in dance from Saint Petersburg University, Russia, in 1996. Since then, she has been with Life Dance
Department, Korea National Sport University. Her main research interests include an introduction to dance and theory of dance composition. 